\title{
Gluon distributions in nucleons and pions at a low resolution scale
}

\author{
H. R. Christiansen* \\ Centro Brasileiro de Pesquisas Físicas, CBPF - DCP \\ Rua Dr. Xavier Sigaud 150, 22290-180, Rio de Janeiro, Brazil \\ and \\ Group of Theoretical Physics, Universidade Católica de Petrópolis \\ Rua Barão de Amazonas 124, 25685-070, Petrópolis, Rio de Janeiro, Brazil \\ and \\ J. Magnin ${ }^{\dagger}$ \\ Depto. de Física, Universidad de los Andes, \\ AA 4976, Santafé de Bogotá, Colombia
}

November 9, 2018

\begin{abstract}
In this paper we study the gluon distribution functions in nucleons and pions at a low resolution $Q^{2}$ scale. This is an important issue since parton densities at low $Q^{2}$ have always been taken as an external input which is adjusted through DGLAP evolution to fit the experimental data at higher scales. Here, in the framework of a model recently developed, it is shown that the hypothetical cloud of neutral pions surrounding nucleons and pions appears to be responsible for the characteristic valence-like gluon distributions needed at the inital low scale. As an additional result, we get the remarkable prediction that neutral and charged pions have different intrinsic sea flavor contents.
\end{abstract}

*e-mail: hugo@cbpf.br

†e-mail: jmagnin@uniandes.edu.co 


\section{Motivation}

Parton distribution functions (pdf) play an important role in particle physics as they describe the internal structure of hadrons in the framework of Quantum Chromodynamics. Pdf are also basic ingredients to calculate cross sections in hadron-hadron and lepton-hadron interactions.

Parton distribution functions have unique characteristics depending on each hadron, which reflect the internal dynamics of the bound state. The pdf, $f_{i}\left(x, Q^{2}\right)$, are interpreted as the probability of finding a parton $i$ (quark or gluon) with a fraction $x$ of the hadron momentum when probed by the momentum transfer $Q^{2}$. The $Q^{2}$ dependence of pdf is succesfully described by the DGLAP [1] evolution equations within perturbative QCD. However, in order to have adequate fits to Deep Inelastic Scattering (DIS) data, initial valence-like distributions at a low resolution scale must be considered even for sea quarks and gluons. This fact has been repeatedly noted by several authors (see e.g. Refs. [2, 3, 4, 5]) but the origin of such initial distributions still remains unclear.

The origin of primordial low scale pdf should be traced back to the internal dynamics of the hadron bound state. Thus they must be related to the confining phase of QCD, which is ultimately the origin of hadrons as bound states of quarks and gluons. In this sense, the sea quark and gluon distributions at a low resolution scale can be related to the idea of the intrinsic sea of quarks and gluons proposed by Brodsky et al. at the beginning of the 80's [6]. In their own words, "intrinsic quarks and gluons exist over a time scale independent of any probe momentum, and are associated with the bound-state hadron dynamics". In contrast, the extrinsic sea of gluons and quarks have a purely perturbative origin, and their distributions show the characteristics of bremsstrahlung and pair production processes leading to the standard DGLAP perturbative QCD evolution.

The above considerations led us to draw a low $Q^{2}$ picture of hadrons in terms of effective quark degrees of freedom interacting among them through intrinsic sea quarks and gluons [7]. The building blocks are the so-called valons which are valence quarks appropriatedly dressed by their extrinsic sea [8]. Within this picture one can represent the hadron wave function as a superposition of hadron-like Fock states which we construct by means of a well-known recombination mechanism [9]. For example, the proton wave function can be written as

$$
|p\rangle=a_{0}\left|p_{\circ}\right\rangle+a_{1}\left|\hat{p}_{\circ} g\right\rangle+\sum_{i} a_{i}\left|B_{i} M_{i}\right\rangle
$$

at some low $Q_{v}^{2}$ scale compatible with the valon picture of the proton. Here $\left|p_{\circ}\right\rangle$ is a pure three valon state [8], and the following terms are hadronic quantum fluctuations which emerge from the (non-perturbative) interaction among valons. The role of these hadronic fluctuations is to dynamically generate the intrinsic sea of quarks and gluons, which is the necessary binding agent in order to have the hadron state. In 
this way, a consistent picture of the low $Q^{2}$ (input) scale of hadrons emerges in which hadrons are formed by constituent quarks plus intrinsic sea quarks and gluons.

It is worth to stress that in modern fits to DIS data, initial non-perturbative sea quark and gluon distributions are taken as an input which is adjusted by DGLAP evolution. In contrast, in our approach they are dynamically generated through the hadronic quantum fluctuations. Furthermore, the old fashioned fits to DIS data, in which all the hadron sea is perturbatively generated, are recovered by restricting the series of eq. (11) to the first term.

This representation of the proton wave function led in a natural way to a $\bar{d} / \bar{u}$ asymmetry in the proton sea which closely describes [10] the most recent experimental data by the E866/NuSea Collaboration [11]. On the same footing, in Ref. [0] a $s-\bar{s}$ asymmetry in the nucleon sea was calculated, qualitatively agreeing with the results of the last global analysis of DIS data 12].

In the following section of this paper, we determine the low $Q^{2}$ non-perturbative gluon distributions in nucleons, using the model introduced in Ref. [7]. In section 3 we explore the consequences of this picture on the non-perturbative structure of charged and neutral pions. Finally, section 4 is devoted to further discussion and conclusions.

\section{Intrinsic gluon distributions}

To start with, let us consider a baryon at some low $Q_{v}^{2}$ scale. At this scale the baryon ground state is formed only by three valons [8]. Quantum fluctuations will generate the non-perturbative $q \bar{q}$ sea. Following Ref. [7], the non-perturbative sea has a twostep origin in our model. In the first step a valon emits a gluon which subsequently decays into a quark-antiquark pair. In the second, such quark and antiquark interact with the valons giving rise to a bound $|M B\rangle$ state. Non-perturbative quark and antiquark distributions are then associated to the in-hadron meson and baryon valon densities.

The emission of a gluon out of a valon is a basic QCD process which can be adequately described in terms of the convolution of the valon distribution, $v(z)$, with the Altarelli-Parisi $P_{g q}(z)$ and $P_{q g}(z)$ splitting functions [1]. In this way, the quark and antiquark initial distributions are given by [7]

$$
q(x)=\bar{q}(x)=N \frac{\alpha_{s t}^{2}\left(Q_{v}^{2}\right)}{(2 \pi)^{2}} \int_{x}^{1} \frac{d y}{y} P_{q g}\left(\frac{x}{y}\right) \int_{y}^{1} \frac{d z}{z} P_{g q}\left(\frac{y}{z}\right) v(z) .
$$

Notice that, as the valon distribution does not depend on $Q^{2}$, the scale dependence in eq. (2) only arises through the strong coupling constant $\alpha_{s t}$. At this stage the scale is fixed to the valon scale, $Q_{v}^{2}$, which is tipically about $1 \mathrm{GeV}^{2}$. Indeed, in Ref. [8] the valon scale was estimated to be of the order of $Q_{v}^{2} \simeq 0.64 \mathrm{GeV}^{2}$ for nucleons. Consequently, pair creation can be safely evaluated in a perturbative way since $\left(\alpha_{s t} / 2 \pi\right)^{2}$ is still sufficiently small. 
The perturbative $v \rightarrow g \rightarrow q \bar{q}$ process is the source of both the extrinsic and intrinsic seas. The difference among them rest on the fact that an intrinsic $q \bar{q}$ pair interacts with the remaining valons while an extrinsic $q \bar{q}$ pair not. In this sense, the extrinsic sea, which is purely perturbative, forms the structure of valons [8].

The second step involves the interaction of such $q \bar{q}$ pair with valons thus giving rise to the $|M B\rangle$ bound state. As they are in the realm of confinement, the interactions of the $q \bar{q}$ pair with valons must be evaluated by means of effective methods. Notice also that, for such interactions take place, the initial (perturbative) $q \bar{q}$ pair must be sufficiently longlived. Since the characteristic lifetime of such a perturbative $q \bar{q}$ pair scales as $1 / m_{q}$, light and strange quarks should be largely available to interact with valons thus producing the $|M B\rangle$ hadronic quantum fluctuations.

Then, assuming that the in-hadron meson and baryon formation arises from mechanisms similar to those at work in the production of real hadrons, we can evaluate the in-hadron meson probability density by using the Das-Hwa recombination approach [9].

In the recombination model, the probability density for the production of a real meson as a function of its fractional momentum is given by the convolution of a two-quark distribution with a suitable recombination function. The two-quark distribution is given in terms of the single-quark distributions of the initial hadron which will be the valence quarks in the final meson. The recombination function is chosen in such a way that it favors the recombination of quarks with similar momentum fractions.

Thus, in our model, the in-hadron meson distributions are given by

$$
P_{M_{i} B_{i}}(x)=\int_{0}^{1} \frac{d y}{y} \int_{0}^{1} \frac{d z}{z} F_{i}(y, z) R(x, y, z),
$$

where

$$
R(x, y, z)=\alpha \frac{y z}{x^{2}} \delta\left(1-\frac{y+z}{x}\right)
$$

is the recombination function [9], and

$$
F_{i}(y, z)=\beta y v(y) z \bar{q}_{i}(z)(1-y-z)^{a}
$$

is the valon-antiquark distribution [10]. In eqs. (3)-(5), $x, y$ and $z$ are the momentum fraction of the in-hadron meson, the valon and the antiquark respectively. The index $i$ runs over different quark flavors, depending on the meson being formed.

Due to momentum conservation, the in-hadron meson and baryon probability densities are not independent but correlated by

$$
P_{M_{i} B_{i}}(x)=P_{B_{i} M_{i}}(1-x),
$$

with an additional correlation in velocity given by

$$
\frac{\left\langle x P_{M_{i} B_{i}}(x)\right\rangle}{m_{M_{i}}}=\frac{\left\langle x P_{B_{i} M_{i}}(x)\right\rangle}{m_{B_{i}}} .
$$


The above constraint, eq. (7), which is needed in order to build a $\left|M_{i} B_{i}\right\rangle$ bound state, fixes the exponent $a$ in eq. (5) [10].

The hadronic fluctuations so far described can be interpreted as the origin of the intrinsic quark-antiquark sea. As a consequence, since the resulting $q$ and $\bar{q}$ sea distributions belong to different hadronic states in the $\left|M_{i} B_{i}\right\rangle$ fluctuation, intrinsic quark and antiquark probability densities in baryons are unequal in a general way.

At this point, a judicious analysis of what fluctuations should be included in an expansion like eq. (1) must be made. For definitness, consider the proton wave function. Taking into account mass values and quantum numbers, the main fluctuations of the proton should be the $\left|\pi^{+} n\right\rangle,\left|\pi^{+} \Delta^{0}\right\rangle$ and $\left|\pi^{-} \Delta^{++}\right\rangle$virtual states, with probabilities $\left|a_{\pi n}\right|^{2}$ and $\left|a_{\pi \Delta}\right|^{2}$ respectively. Differences between the $\left|\pi^{+} \Delta^{0}\right\rangle$ and $\left|\pi^{-} \Delta^{++}\right\rangle$ probabilities are taken into account by Clebsh-Gordan coefficients which ensure the correct global isospin of the fluctuation. Thus, we obtain $\frac{1}{6}\left|a_{\pi \Delta}\right|^{2}$ and $\frac{1}{2}\left|a_{\pi \Delta}\right|^{2}$ for $\left|\pi^{+} \Delta^{0}\right\rangle$ and $\left|\pi^{-} \Delta^{++}\right\rangle$respectively. On the other hand, the probability of the $\left|\pi^{+} n\right\rangle$ bound-state is $\frac{2}{3}\left|a_{\pi n}\right|^{2}$. The coefficients $\left|a_{\pi n}\right|^{2}$ and $\left|a_{\pi \Delta}\right|^{2}$ are given by $(N \alpha \beta)_{\pi n}$ and $(N \alpha \beta)_{\pi \Delta}$ respectively. Their numerical values result from comparison with experimental data. Fluctuations like $|\rho N\rangle,|\rho \Delta\rangle$, etc. which could contribute, for instance, to the $\bar{d}-\bar{u}$ asymmetry in the proton, are far off-shell 1 and can be safely neglected.

Remarkably, as shown in Ref. [10], this scheme leads to a $\bar{d} / \bar{u}$ asymmetry in the proton which closely describes the experimental data by the E866 Collaboration [1]. Including fluctuations to Kaon-Hyperon states, $|K H\rangle$, a $s-\bar{s}$ asymmetry in the proton sea arises [7] which qualitatively agrees with results from a recent global analysis of DIS data [12].

Fluctuations of the proton to $\left|\pi^{0} p\right\rangle$ and $\left|\pi^{0} \Delta^{+}\right\rangle$states do not contribute to the intrinsic quark and antiquark structure. The reason is that the formation of a $\pi^{0}$ in-proton state must be inhibited due to its neutral flavor structure $u \bar{u}-d \bar{d}$. This happens because $v_{q} \bar{q}$ objects can annihilate rapidly into a gluon while $v_{q} \bar{q}^{\prime}$ cannot $\left(q^{\prime}\right.$ is a quark of different flavor to the $q$ one. See Fig. 1). Notice also that an unflavored object like a $v_{q} \bar{q}$ pair has itself the quantum numbers of a gluon. Thus, a hypothetical $\left|\pi^{0} p\right\rangle$ fluctuation does not contribute to the sum over $\left|M_{i} B_{i}\right\rangle$ in the RHS of eq. (1) but to the second term, $\left|\hat{p}_{\circ} g\right\rangle$, providing a source of valence-like gluons in the proton. The proton-like object accompanying the non-perturbative gluon in the $\left|\hat{p}_{\circ} g\right\rangle$ fluctuation must have the same flavor structure than the $p_{\circ}$ in the $\left|p_{\circ}\right\rangle$ state. However, as far as the gluon is in a color octect state, the $\hat{p}_{\circ}$ must be colored. It is worth noting that, on general grounds, hadrons in a $|M B\rangle$ fluctuation must be colored. However, they can be identified with real hadrons regarding other quantum numbers like as flavor, isospin, etc.

The time scale over which both the $\left|\pi^{+} n\right\rangle$ and the $\left|\hat{p}_{\circ} g\right\rangle$ fluctuations exist should be approximately the same. In fact, the characteristic lifetime of a $|M B\rangle$ fluctuation is proportional to $1 / \Delta E$, where $\Delta E$ is the energy difference between the $|M B\rangle$ and

\footnotetext{
${ }^{1}$ Note that these are even more suppressed than strange, $|K H\rangle$, fluctuations.
} 
the proton states in an infinite momentum frame. Thus, for a generic $|M B\rangle$ state we have

$$
\tau_{|M B\rangle} \sim \frac{1}{\Delta E}=\frac{2 P}{\left[\frac{\hat{m}_{M}^{2}}{x_{M}}+\frac{\hat{m}_{B}^{2}}{x_{B}}-m_{p}^{2}\right]},
$$

where $P$ is the momentum of the proton in the infinite momentum frame, $m_{p}$ is the proton mass, and $x_{M}$ and $x_{B}$ are the momentum fractions carried by the meson and baryon in the fluctuation. $\hat{m}_{M, B}^{2}=m_{M, B}^{2}+k_{T}^{2}$ is the transverse masses squared of virtual hadrons in the fluctuation. Given the smallness of the pion mass, we can assume that in-nucleon pions and non-perturbative gluons have similar transverse masses, then the characteristic lifetimes of the $\left|\pi^{+} n\right\rangle$ and $\left|\hat{p}_{\circ} g\right\rangle$ fluctuations must be approximately the same.

In this approach, the shape of the non-perturbative gluon coming from the $v_{q} \bar{q}$ pairing above described can be estimated by using the recombination model. Actually, as the origin of the non-perturbative gluon is the recombination of a valon with an antiquark of the same flavor, the momentum distribution of intrinsic gluons in the $\left|\hat{p}_{\circ} g\right\rangle$ fluctuation is simply given by

$$
\begin{aligned}
g^{N P}\left(x, Q_{v}^{2}\right) & =\left|a_{1}\right|^{2} P_{p g}\left(x, Q_{v}^{2}\right) \\
& \equiv\left|a_{1}\right|^{2} \frac{(1-x)^{12.9}}{x} \int_{0}^{x} d y y \bar{q}(y)(y-x) v_{N q}(y-x)
\end{aligned}
$$

where $\bar{q}(x)$ is given by eq. (2) and $v_{N q}(x)$ is the distribution of the $q$-flavored valon in the nucleon given by 8

$$
v_{N q}(x)=\frac{105}{32} \sqrt{x}(1-x)^{2} .
$$

Coefficients $N, \alpha$, and $\beta$; coming from the definition of the initial perturbative $\bar{q}$, the recombination function and the valon-antiquark distribution in eqs. (2), (41) and (5) respectively; were included in the definition of $\left|a_{1}\right|^{2}$, the probability of the fluctuation. The intrinsic gluon probability distribution, $P_{p g}\left(x, Q_{v}^{2}\right)$, is accordingly normalized to unity.

The intrinsic gluon probability density in the $\left|\Delta^{+} g\right\rangle$ fluctuation can be estimated on similar basis. However, this fluctuation should be suppressed with respect to the $\left|\hat{p}_{\circ} g\right\rangle$ one.

Another way to have a proton fluctuation containing a proton-like object and an unflavored neutral meson would be through the self-recombination of the $q-$ $\bar{q}$ pair produced by the gluon splitting of eq. (2) (see Fig. 2). In this case, the unflavored neutral meson must be a vector meson like a $\rho^{0}$ or $\omega$. This is necessary to preserve the vectorial character of the initial perturbative gluon. However, this kind of fluctuations, consisting of a disconnected $\rho^{0}$ or $\omega$ and a $\hat{p}_{\circ}$, are strongly suppressed by the OZI rule. 
In Fig. 3, the intrinsic gluon distribution at the valon scale given by eq. (9) is compared to the initial GRV-94 HO [2] gluon distribution and the valence gluon distribution calculated in a Monte Carlo based model of the proton [5] If.

\section{Non-perturbative structure of pions}

Similar mechanisms should be at work in other physical hadrons, like pions. Indeed, if we expand the pion wave function as

$$
\left|\pi^{ \pm, 0}\right\rangle=b_{0}\left|\pi_{\circ}^{ \pm, 0}\right\rangle+b_{1}\left|\hat{\pi}_{\circ}^{ \pm, 0} g\right\rangle+\sum_{i} b_{i}\left|M_{i} M_{i}^{\prime}\right\rangle
$$

we can identify the would be $\left|\hat{\pi}_{\circ}^{ \pm, 0} \pi^{0}\right\rangle$ fluctuation with the $\left|\hat{\pi}_{\circ}^{ \pm, 0} g\right\rangle$ one, as we made for nucleons. In this way, the intrinsic gluon contribution to the pion low $Q^{2}$ structure is given by a similar expression to that of eq. (9),

$$
\begin{aligned}
g_{\pi}^{N P}\left(x, Q_{v}^{2}\right) & =\left|b_{1}\right|^{2} P_{\pi g}\left(x, Q_{v}^{2}\right) \\
& =\left|b_{1}\right|^{2} \frac{(1-x)}{x} \int_{0}^{x} d y y \bar{q}_{\pi}(y)(y-x) v_{\Pi}(y-x),
\end{aligned}
$$

where $\bar{q}_{\pi}(x)$ is an antiquark distribution analogous to $\bar{q}(x)$ in eq. (2) but with a valon distribution for pions $v_{\Pi}(x)=1$, as given in [8]. Regarding the exponent related to velocity correlation, it turns out to be simply $a=1$. For the valon scale in pions we have used the same value than for nucleons, $Q_{v}^{2} \simeq 0.64 \mathrm{GeV}^{2}$.

As explained above, a $v_{q} \bar{q}$ pair should recombine into a gluon instead of forming a neutral pion structure. Thus the same conclusions drawn for nucleons hold for fluctuations of the pion into a Fock state containing the initial pion plus a neutral pion-like object.

In Fig. 1 , the $g_{\pi}^{N P}\left(x, Q_{v}^{2}\right)$ predicted by the model is shown, and compared to the GRV-P HO [13 distribution and the gluon distribution in pions obtained in Ref. [5]. It is interesting to note that our model predicts intrinsic gluon distributions carrying more average momentum in pions than in nucleons. This is a consequence of the fact that the $v_{q} \bar{q}$ pair giving rise to the intrinsic gluon carries more average momentum in a $\left|\hat{\pi}_{\circ}^{ \pm, 0} g\right\rangle$ fluctuation than in a $\left|\hat{p}_{\circ} g\right\rangle$ fluctuation.

The fact that pions do not fluctuate into $\left|\hat{\pi}_{0}^{ \pm, 0} \pi^{0}\right\rangle$ states but into $\left|\hat{\pi}_{0}^{ \pm, 0} \mathrm{~g}\right\rangle$ ones has additional consequences on their low $Q^{2}$ scale structure. For example, for charged

\footnotetext{
${ }^{2}$ In [5], a model for hadrons is proposed in which primordial pdf corresponding to valence quarks and gluons are assumed to have Gaussian distributions with widths fixed from experimental data. These initial pdf are then complemented with contributions coming from $|M B\rangle$ fluctuations. In this model, intrinsic gluons are supposed to be present from the very beginning. In our model we are proposing a dynamical mechanism for their generation. This is the main difference between these two approaches.
} 
pions the first contribution to the sum in the RHS of eq. (11) arises from the $\left|K^{+} \bar{K}^{0}\right\rangle$ $\left(\left|K^{-} K^{0}\right\rangle\right)$ fluctuation of the $\left|\pi_{\circ}^{+}\right\rangle\left(\left|\pi_{\circ}^{-}\right\rangle\right)$state. Thus,

$$
\begin{aligned}
& \left|\pi^{+}\right\rangle=b_{0}\left|\pi_{\circ}^{+}\right\rangle+b_{1}\left|\pi_{\circ}^{+} g\right\rangle+b_{3}\left|K^{+} \bar{K}^{0}\right\rangle+\ldots \\
& \left|\pi^{-}\right\rangle=b_{0}\left|\pi_{\circ}^{-}\right\rangle+b_{1}\left|\pi_{\circ}^{-} g\right\rangle+b_{3}\left|K^{-} K^{0}\right\rangle+\ldots
\end{aligned}
$$

Then the first contribution to the intrinsic $q \bar{q}$ sea arises in the strange sector and there are no $u \bar{u}$ and $d \bar{d}$ intrinsic seas in charged pions. The structure of charged pions at the low $Q_{v}^{2}$ scale is thus given by

$$
\begin{aligned}
v_{q / \pi^{ \pm}}\left(x, Q_{v}^{2}\right)= & \left|b_{0}\right|^{2} v_{\Pi}(x)+\left|b_{1}\right|^{2} \int_{x}^{1} \frac{d y}{y} P_{g \pi}(y) v_{\Pi}\left(\frac{x}{y}\right) \\
& +\left|b_{3}\right|^{2} \int_{x}^{1} \frac{d y}{y} P_{K K}(y) v_{K q}\left(\frac{x}{y}\right) \\
s_{s}\left(x, Q_{v}^{2}\right)= & \bar{s}_{s}\left(x, Q_{v}^{2}\right)=\left|b_{3}\right|^{2} \int_{x}^{1} \frac{d y}{y} P_{K K}(y) v_{K s}\left(\frac{x}{y}\right),
\end{aligned}
$$

where $v_{q / \pi^{ \pm}}$are the resulting pion valence quark densities, $v_{K q}$ and $v_{K s}$ are the light and strange valon distributions in Kaons, and $s_{s}=\bar{s}_{s}$ the non-perturbative strange quark distributions in charged pions. $P_{K K}$ is the probability density of a Kaon inside a pion and $P_{g \pi}(x)=P_{\pi g}(1-x)$ is the charged pion distribution in the $\left|\hat{\pi}_{\circ}^{ \pm} g\right\rangle$ fluctuation. The hadronic distributions inside pions, $P_{\pi g}$ and $P_{K K}$, are given by similar formulas to those of eqs. (3)-(5).

It is interesting to note that light quarks in a $|K K\rangle$ fluctuation contribute to the charged pion low $Q^{2}$ valence densities but not to their intrinsic sea distributions. This is because although there are non-perturbative contributions to the light quark distributions in charged pions, they appear in the $\bar{u}(u)$ and $d(\bar{d})$ sectors but not in the $u(\bar{u})$ and $\bar{d}(d)$ for the $\pi^{-}\left(\pi^{+}\right)$respectively ${ }^{\text {f }}$. In turn, for neutral pions the hadronic Fock state expansion has the form

$$
\left|\pi^{0}\right\rangle=b_{0}\left|\pi_{\circ}^{0}\right\rangle+b_{1}\left|\hat{\pi}_{\circ}^{0} g\right\rangle+b_{2}\left|\pi^{-} \pi^{+}\right\rangle+\frac{b_{3}}{\sqrt{2}}\left[\left|K^{-} K^{+}\right\rangle-\left|K^{0} \bar{K}^{0}\right\rangle\right]+\ldots
$$

Then, by analogy with eqs. (15), we can define

$$
\begin{aligned}
v_{q / \pi^{0}}\left(x, Q_{v}^{2}\right)= & \frac{1}{2}\left|b_{0}\right|^{2} v_{\Pi}(x)+\frac{1}{2}\left|b_{1}\right|^{2} \int_{x}^{1} \frac{d y}{y} P_{g \pi}(y) v_{\Pi}\left(\frac{x}{y}\right) \\
& +\frac{\left|b_{3}\right|^{2}}{2} \int_{x}^{1} \frac{d y}{y} P_{K K}(y) v_{K q}\left(\frac{x}{y}\right)
\end{aligned}
$$

\footnotetext{
${ }^{3}$ Recall the flavor structure of the particles involved: $\pi^{+}(u \bar{d}) \rightarrow K^{+}(u \bar{s}) \bar{K}^{0}(s \bar{d})$ and $\pi^{-}(\bar{u} d) \rightarrow$ $K^{-}(\bar{u} s) K^{0}(\bar{s} d)$.
} 
for the valence quark densities at the low $Q_{v}^{2}$ scale, and

$$
\begin{aligned}
s_{u / \pi^{0}}\left(x, Q_{v}^{2}\right) & =s_{\bar{u} / \pi^{0}}\left(x, Q_{v}^{2}\right)=s_{d / \pi^{0}}\left(x, Q_{v}^{2}\right)=s_{\bar{d} / \pi^{0}}\left(x, Q_{v}^{2}\right) \\
& =\left|b_{2}\right|^{2} \int_{x}^{1} \frac{d y}{y} P_{\pi \pi}(y) v_{\Pi}\left(\frac{x}{y}\right) \\
s_{s}\left(x, Q_{v}^{2}\right) & =\bar{s}_{s}\left(x, Q_{v}^{2}\right)=\left|b_{3}\right|^{2} \int_{x}^{1} \frac{d y}{y} P_{K K}(y) v_{K s}\left(\frac{x}{y}\right),
\end{aligned}
$$

for the intrinsic up, down and strange seas. Gluon distributions are given by eq. (12) for both neutral and charged pions.

It should be noted that, although considering the $s_{q / \pi^{0}}$ densities as part of the intrinsic light sea or part of the valence densities in the $\pi^{0}$ is a matter of convention, the low $Q_{v}^{2}$ structure of the $\pi^{0}$ is different to the structure of charged pions. The difference is precisely given by the contribution of the $\left|\pi^{-} \pi^{+}\right\rangle$fluctuation which can only occur in a $\pi^{0}$ state.

As a final result, notice that the intrinsic quark-antiquark sea of pions turns out to be symmetric as a consequence of the hadronic structure of the fluctuations. This is in contrast to the tipically unequal intrinsic quark and antiquark distributions of the nucleon (see e.g. [0] and Refs. therein).

\section{Conclusions}

In this paper we have analysed some important consequences of making a hadronic Fock state expansion of the nucleon and pion low $Q^{2}$ wave-functions out of a novel mechanism for generating the cloud. We have shown that within such scheme it is possible to generate not only non-perturbative quark-antiquark distributions but also the gluon sea needed at the low $Q^{2}$ starting scale for DGLAP evolution.

These non-pertubative quarks and gluons are responsible for the bound nature of any hadron state, as they bring about the interactions between valence quarks. The non-perturbative quarks and gluons can be consistently identified with the so called intrinsic sea, in contrast to the extrinsic sea. On the other hand, the extrinsic sea is perturbatively generated by the probe momentum $Q^{2}$, and is part of the own structure of valons, as discussed long time ago by Hwa [8].

In this sense our approach leads to a unification of two different pictures of the hadron structure; namely, the early picture of (non-interacting) valons [8], and the intrinsic sea idea of Brodsky et al. [6], which provides the binding agent for the bound hadron state. On the other hand, our approach allows a full representation of the non-perturbative processes giving rise to hadronic quantum fluctuations. This fluctuations are due to the perturbative production of a $q \bar{q}$ pair which recombines with the remaining valons. Thus a connection between the physics of hadronic reactions 
and that of hadronic fluctuations is established through the well known recombination mechanism.

A remarkable feature of the approach is that neutral pion fluctuations are here inhibited and, in turn, non-perturbative gluons take place. The reason is that neutral unflavored structures like the initial $v_{q} \bar{q}$ objects, are more likely to recombine rapidly into gluons than into neutral pions, in contrast to flavored structures like $v_{q} \bar{q}^{\prime}$ which cannot do so. Thus, the hypothetical cloud of quantum fluctuations like $\left|\hat{p}_{\circ} \pi^{0}\right\rangle$ does not contribute to the sum over $\left|B_{i} M_{i}\right\rangle$ in the RHS of eq. (四) but to the second term, $\left|\hat{p}_{\circ} g\right\rangle$, providing the source of valence-like gluons in the proton.

Thus, within our scheme not only intrinsic quarks and antiquarks but also gluons are generated through quantum fluctuations of the low $Q^{2}$ hadron ground state.

Concerning pions, we have calculated their quark-antiquark and gluon distributions at low $Q^{2}$. We have also shown that the non-perturbative structure of charged and neutral pions are different. The difference arises from the $\left|\pi^{+} \pi^{-}\right\rangle$fluctuation appearing in the hadronic Fock state expansion of the $\pi^{0}$ wave-function but not in the charged pion ones. Finally, we have shown that the pionic intrinsic quark and antiquark distributions are symmetric, as a result of the specific features of its quantum fluctuations. This is in contrast to the structure of generic baryons which have asymmetric intrinsic quark and antiquark distributions [7, 10]. However, it should be noted that for mesons containing a light and a heavier valence quark, the situation is different and the intrinsic quark-antiquark sea must be asymmetric [14].

Summarizing, we have proposed a possible scenario for the origin of the valencelike sea quark and gluon distributions nedeed at the low (input) scale in order to describe the experimental DIS data for nucleons and pions. We have also discussed the low scale structure of charged pions and shown that, besides valence quarks, the model predicts only gluon and strange intrinsic sea distributions as a suitable low $Q^{2}$ starting point for perturbative DGLAP evolution. On the other hand, for neutral pions, intrinsic light quark-antiquark distribution have to be considered as well. This signals a remarkable difference between the non-perturbative structure of neutral and charged pions.

\section{Acknowledgments}

We acknowledge R. Vogt for useful comments. J.M. is partially supported by COLCIENCIAS, the Colombian Agency for Science and Technology, under Contract No. 242-99.

\section{References}


[1] G. Altarelli and G. Parisi, Nucl. Phys. B126, 298 (1977); Yu. L. Dokshitzer, Sov. Phys. JETP 46, 641 (1977); V.N. Gribov and L.N. Lipatov, Sov.J. Nucl. Phys. 15, 428 (1972).

[2] M. Glück, E. Reya and A. Vogt, Z. Phys. C53, 127 (1992), C67, 433 (1995).

[3] J. Botts et al., Phys. Lett. B304, 159 (1993); H.L. Lai et al., Phys. Rev. D51, 4763 (1995).

[4] A.D. Martin, W.J. Stirling and R.G. Roberts, Phys. Lett. B354, 155 (1995).

[5] A. Edin and G. Ingelman, Phys. Lett. B432, 402 (1998).

[6] S.J. Brodsky, C. Peterson and N. Sakai, Phys. Rev. D23, 2745 (1981).

[7] H.R. Christiansen and J. Magnin, Phys. Lett. B445, 8 (1998).

[8] R.C. Hwa, Phys. Rev. D22, 1593 (1980).

[9] K.P. Das and R.C. Hwa, Phys. Lett B68, 459 (1977).

[10] J. Magnin and H.R. Christiansen, Phys. Rev. D61, 054006 (2000).

[11] E.A. Hawker et al., Phys. Rev. Lett. 80, 3715 (1998); J.C. Peng et al., Phys. Rev. D58, 092004 (1998).

[12] V. Barone, C. Pascaud and F. Zomer, Eur. Phys. J. C12, 243 (2000).

[13] M. Glück, E. Reya and A. Vogt, Z. Phys. C53, 651 (1992).

[14] C. Avila, J. Magnin and J.C. Sanabria, hep-ph/0005287. 

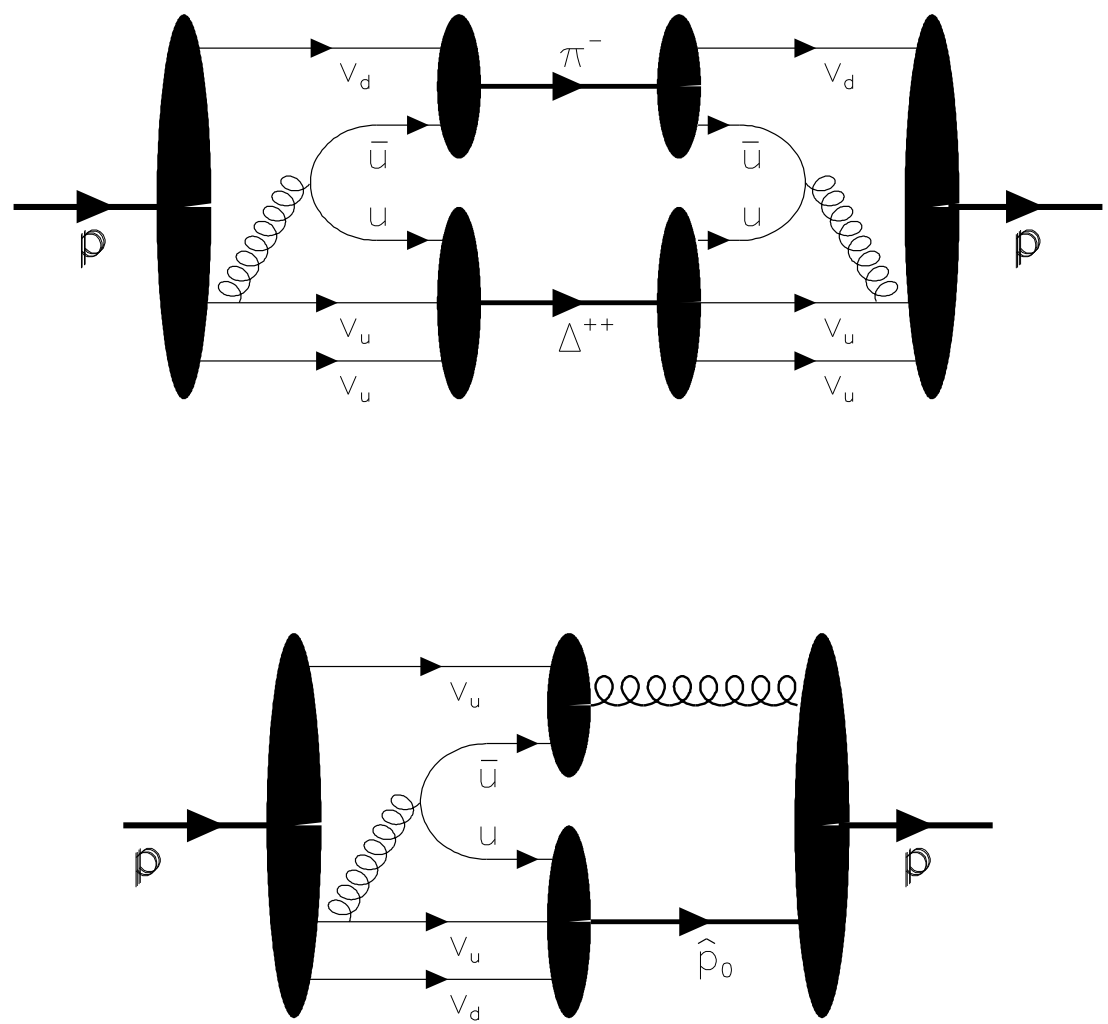

Figure 1: Diagramatic representation of the process giving rise to a $\left|\pi^{-} \Delta^{++}\right\rangle$fluctuation of the proton (upper). Process leading to the generation of intrinsic gluons through the recombination of a $v_{q} \bar{q}$ pair into a gluon (lower). 


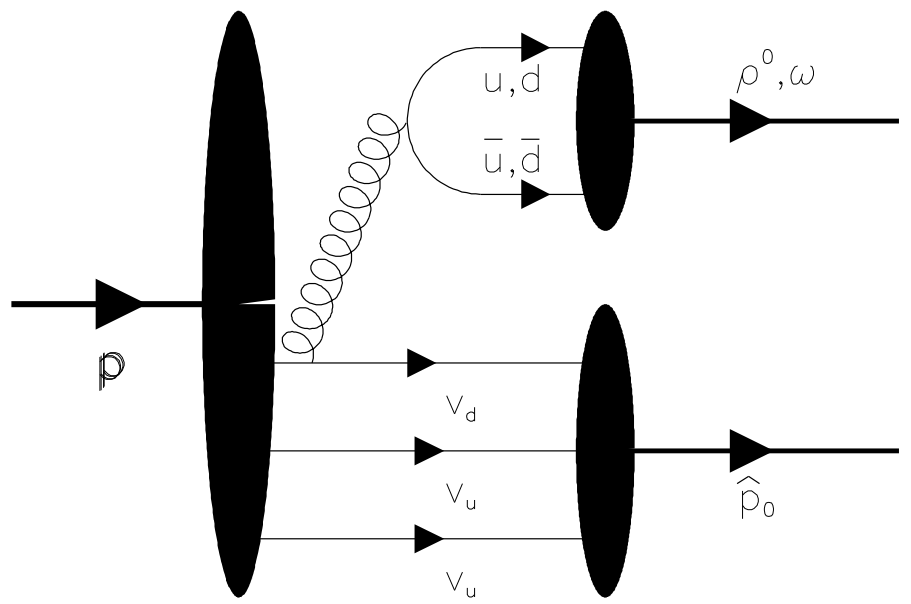

Figure 2: OZI rule suppressed $\left|\rho^{0}(\omega) \hat{p}_{\circ}\right\rangle$ fluctuation. 


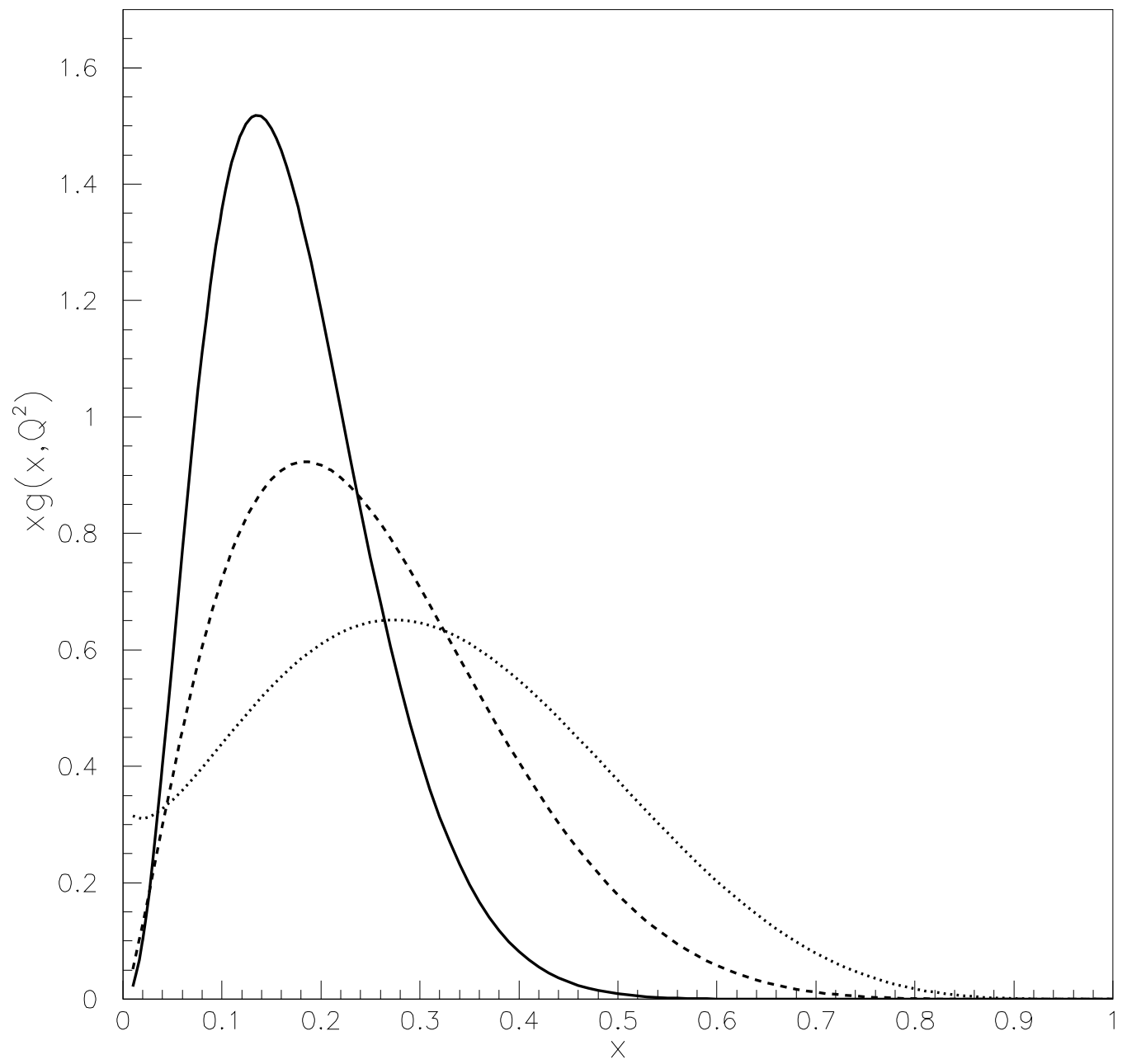

Figure 3: Intrinsic gluon distribution for nucleons predicted by the model at $Q_{v}^{2} \simeq 0.64$ $\mathrm{GeV}^{2}$ (solid line) compared to the valence gluon distribution given by the model of Ref. [5] at $Q_{0}^{2} \sim 1 \mathrm{GeV}^{2}$ (dashed line) and the initial GRV-94 HO gluon distribution [2] at $Q_{0}^{2}=0.4 \mathrm{GeV}^{2}$ (point line). Model curves were normalized to the value of the integral over $x$ of the GRV-HO gluon distribution. 


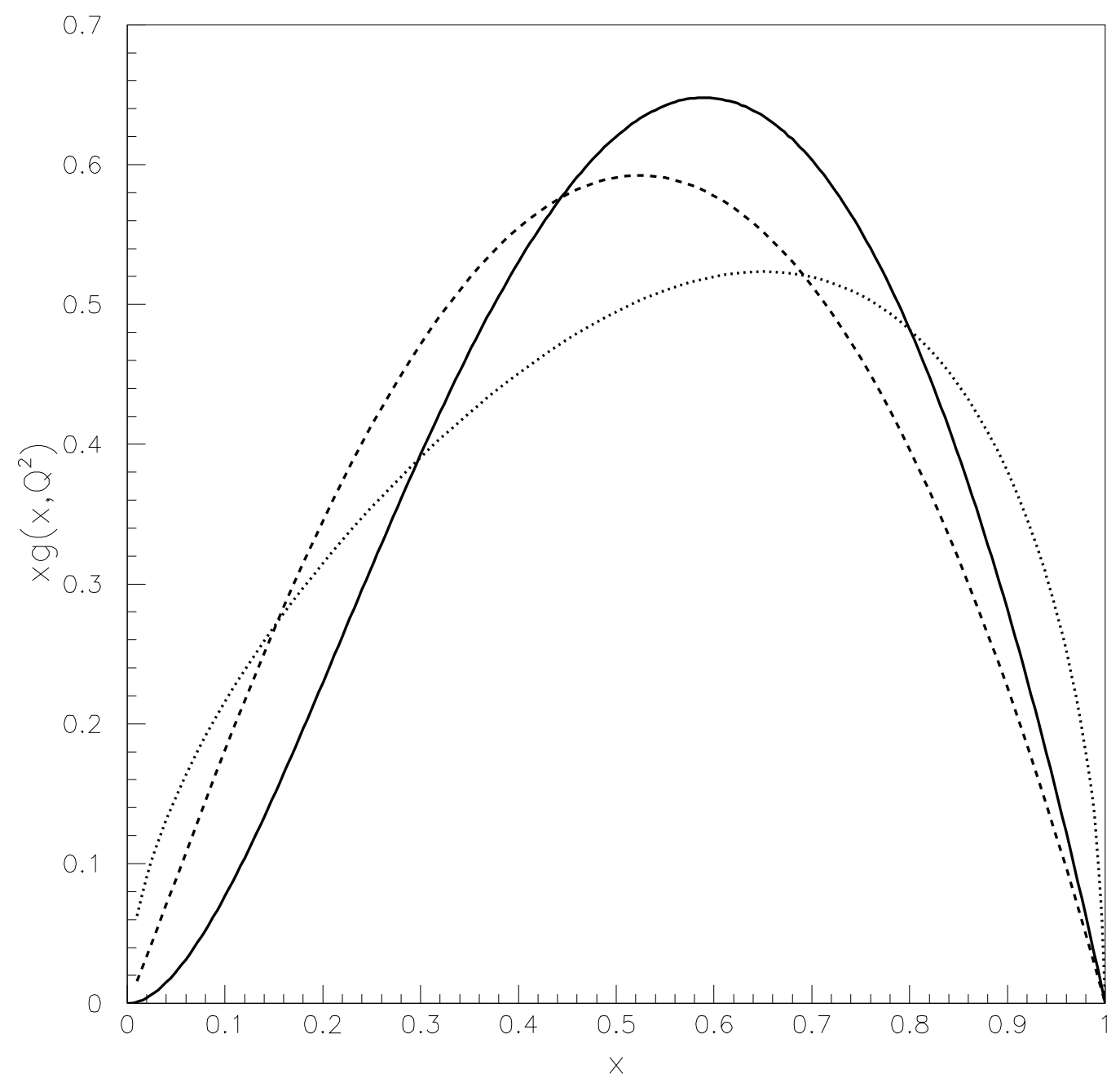

Figure 4: Intrinsic gluon distribution for pions predicted by the model at $Q_{v}^{2} \simeq 0.64$ $\mathrm{GeV}^{2}$ (solid line) compared to the valence gluon distribution given by the model of Ref. [5] at some hadronic low $Q_{0}^{2}$ scale (dashed line) and the initial GRV-P HO gluon distribution 13 at $Q_{0}^{2}=0.3 \mathrm{GeV}^{2}$ (point line). Model curves were normalized to the value of the integral over $x$ of the GRV-P HO gluon distribution. 